\title{
An Emerging Study in Augmented Reality \& Geographical Information System
}

\author{
Jonathan Rodriguez and Ching-Yu Huang
}

\begin{abstract}
Augmented Reality (AR) and Geographic Information System (GIS) can be applied in various areas. They can be utilized to provide information on identifying the environments. Since most of the colleges don't offer AR and GIS courses, this paper presents an independent study framework about how college students can learn AR, GIS, Database, mobile app development through the emerging independent study. It is very important to know how Geolocation can be used to make very dynamic applications that users can interact with based on their location. This Independent Study framework will be using many new web and mobile technologies that are open-sourced. The purpose of this study is to demonstrate the usefulness and the benefits of knowledge on GIS/AR.
\end{abstract}

Index Terms-MongoDB, angularjs, google maps, ionic framework, geolocation, hybrid mobile application.

\section{INTRODUCTION}

Geographic Information System (GIS) is rooted in intellectual practices, populated by data and powered by mathematical analysis. A survey conducted by Schuurman [1] suggested that currently, the main use of GIS is for spatial analysis, predictive modelling, cartography and visualization. The SI Industry, also known as the GIS industry, is a rapidly growing industry. GIS maps the exact location and survey coordinates of an object in space to provide answer to queries using a computer system [2].

Many popular applications like PokemonGO and Uber have great success with their brand as they have geo-based products. Users experience more engagement with the product when their location depends on it. This new trend has exposed the possibilities of geo-based apps and services as they have the most active users. Many of this geo-based apps have made a social impact with their users as they need to go out and move around to get the most out of the app. It has also allowed the users to be more active physically which is a great initiative to be more connected with the world.

As these geo-based apps gain popularity among users, lawmakers are eyeing geolocation apps as there are many privacy concerns have arisen regarding user tracking. Today, GPS location-aware smart phones and other devices collect enormous amounts of data about where people go and what they do. This information can be aggregated with other information to determine 'who they are' with precision and accuracy [3].

As geolocation is becoming an important component of daily lives (Ex. Navigation devices), geolocation will face a raft of new regulations. To avoid leaking consumer data and protecting their privacy, greater protection of the data should be accounted for.

This independent has helped the understanding of why geo-based systems are so popular nowadays and their usefulness for users. With the end product being a location based system which may be used for building navigation or even open house navigation/information for universities, this system could greatly benefit communities that need geo-based systems to help people learn the advantages and possibilities of having location as a main factor for an application.

\section{RELATED WORK}

Typically, geolocation apps do two things: They report your location to other users, and they associate real-world locations (such as restaurants and events) to your location. Geolocation apps that run on mobile devices provide a richer experience than those that run on desktop PCs because the relevant data you send and receive changes as your location changes [4].

To become familiar with the topic of GIS and AR, some resources that have greatly improved the skills needed for this project are the Google Maps API [5]. To store objects like places, buildings, points, etc., Google Maps greatly helps with understanding how these objects are made and stored in the database schema.

Some products that are related to this specific study is Foursquare. With this geolocation service, users are able to check in to cafés, bars, restaurants, and pretty much anyplace. Aside from the social impact of checking in to a place when you're actually there, it empowers users to collect stamps/badges to track progress of places visited, and overall keeping the consumer engaged with the application.

Hackathon participation has also increased knowledge of the topic as it has allowed to quickly iterate concepts to proof the potential of geolocation applications. Hackathon experience has greatly increased quick adaptation of new concepts such as GIS/AR, and database schemas for geographical information which are used in this study.

\section{SYSTEM ARCHITECTURE}

Jonathan Rodriguez was with School of Computer Science at Kean University, Union, NJ, USA (e-mail: rodrjon1 @kean.edu).

Ching-Yu Huang is with School of Computer Science at Kean University, Union, NJ, USA (e-mail: chuang@kean.edu).
In order to visualize the location and other information, a front-end Graphic User Interface (GUI) is required for the 
system. Since the system is designed for portable device such as smartphone, a mobile app is necessary to be developed. The database is the back-end to keep the building coordinates, event information, images and other information. The middle-end is to handle the data upload from front-end and transferring between front-end and back-end.

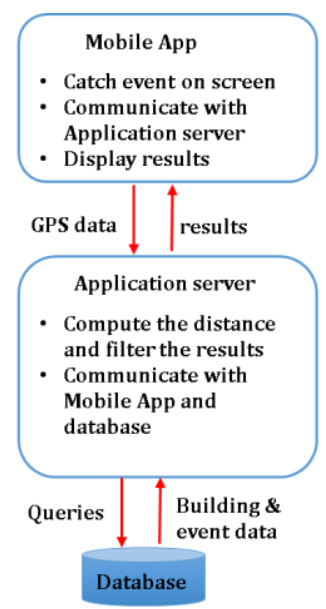

Fig. 1. System architecture.

\section{A. Frontend: Mobile App}

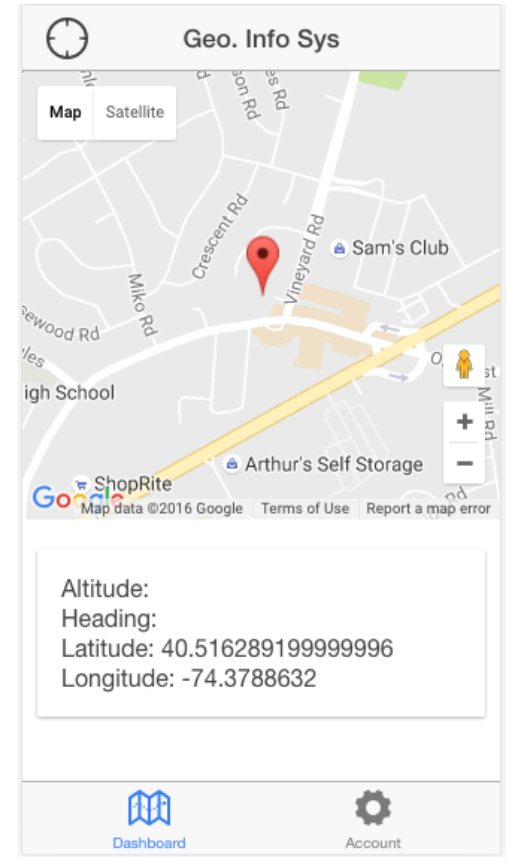

Fig. 2. Automatically check user's location.

The GUI for this application will contain the current location of the user and will display current information about the place they are in (building, classroom, etc.). As a user moves from a building to another or to another classroom, the main view will update with current information about their location, with interactive links and videos that will show the users more information about their location, as shown in Fig. 2.

The mobile app will read the GPS location from the smartphone device and pass the altitude, orientation, latitude, longitude and time information to the application at the middle-end. The Latitude: 40.516289 and Longitude: -
74.378863 indicates the GPS location of the red marker shown on Fig. 2. Since the mobile app uses Google Map API, user can switch to Street View by clicking on the "people icon" at left, or switch to Satellite View by clicking on the "Satellite" at the right top.

To insert these objects to our database, a portal will be built to submit records. A seamless UI will be designed for easy interaction and administrators will be able to submit buildings information alongside with specifics like classroom schedules, special events, and videos about the department. The Google Maps API is used to get the actual coordinates of buildings for submission, as shown in Fig. 3.

It is important to understand that the GPS information might be not accurate in general inside the building [6], [7]. Therefore, it is difficult to precisely identify the location inside a building and display correct event information for each floor and room. So, it might be better to just show the events for the entire building as shown in Fig. 4. Especially, each floor might have different heights, for example the first floor of NAAB building is much taller than the $2^{\text {nd }}$ floor as shown in Fig. 5 in addition to the GPS altitude values inside the building could be not very accurate.

Since the GPS coordinates of the NAAB building boundary are not available, we have to manually mark and read the GPS coordinates on Google map [8] as shown in Fig. 6, and store the information into the database.

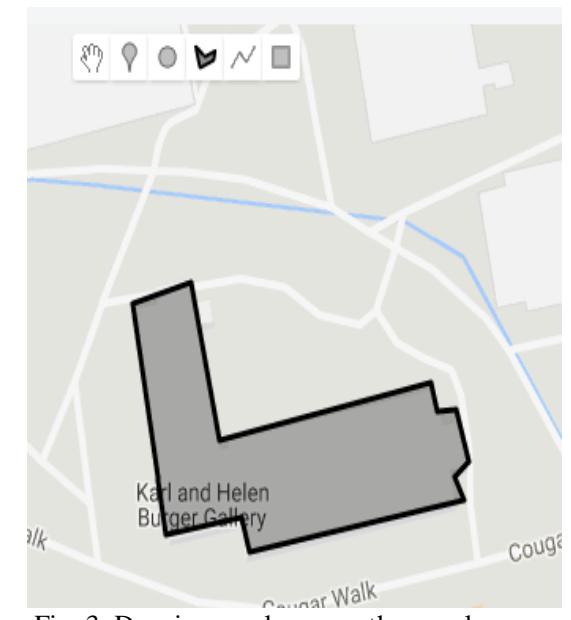

Fig. 3. Drawing a polygon on the google maps.

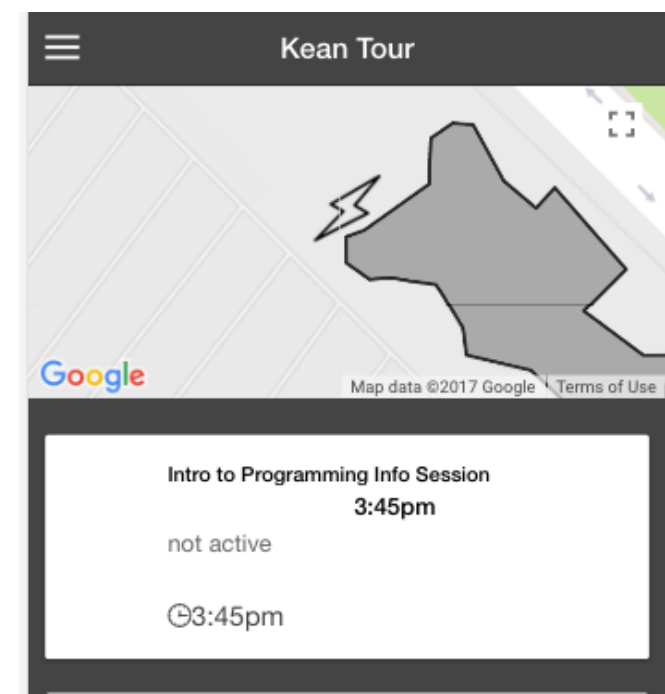

Fig. 4. The polygon of NAAB building on Google map. 


\section{B. Middle-End: Application Server}

The application server is written in Javascript and is used in par with AngularJS and Ionic Framework. These are new, open-sourced technologies that will help build a robust app front-end and server to handle the amount of computations that will be made.

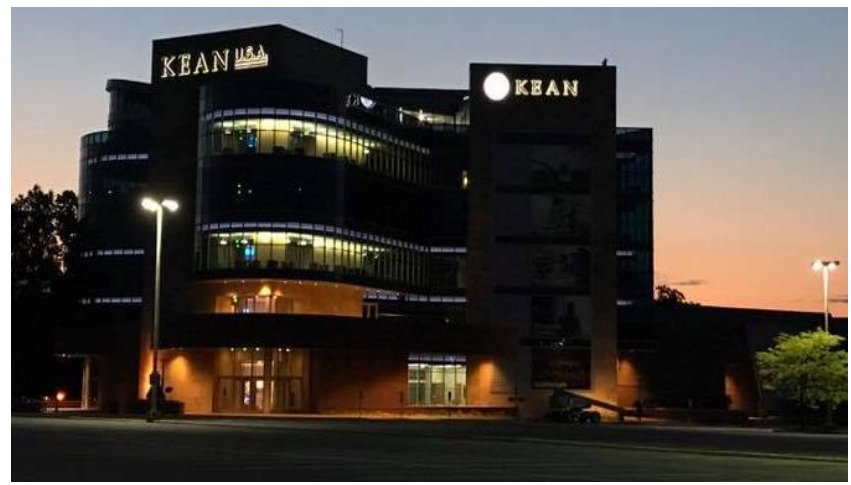

Fig. 5. The NAAB building at Kean University.

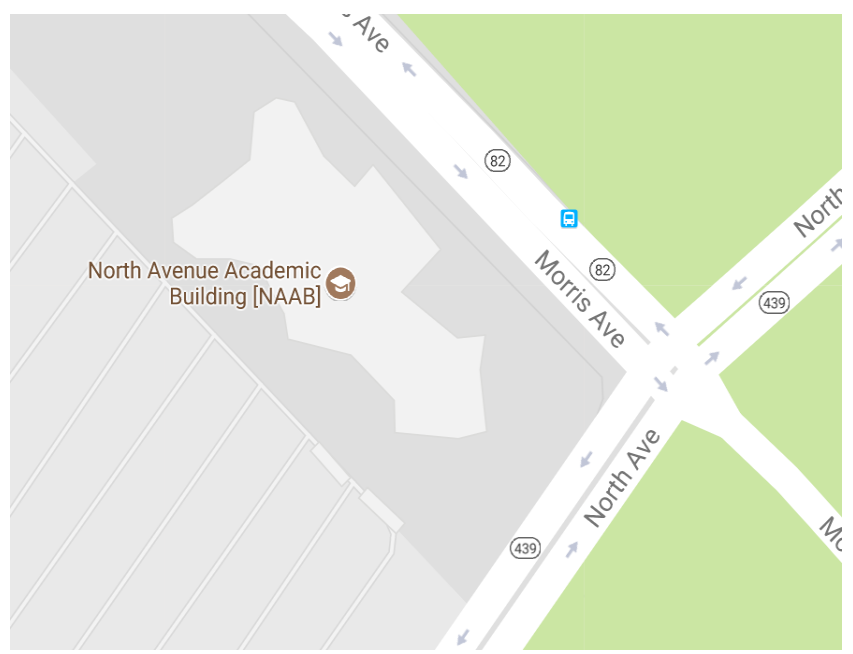

Fig. 6. The NAAB building displayed on a browser based on www.googlemap.com.

The application requires a lot of computations in order for the user to get updated geographic information and interacting with the app. Understanding these computations and how to decrease the amount of computations enhanced math knowledge and overall computer science foundation.

Some crucial fields like the GPS coordinates will be stored on the database, these coordinates come as points or paired up points (Polygon). These points are a combination of latitude and longitude pairs, as shown similar to a picture EXIF information extraction, shown in Fig. 6.

\begin{tabular}{|c|c|}
\hline \multicolumn{2}{|l|}{ GPS information: } \\
\hline GPSLatitudePef & $\mathrm{N}$ \\
\hline GPSLatitude & $40 \quad 40 \quad 45.36(40.679267)$ \\
\hline GPSLongitudeRef & $w$ \\
\hline GPSLongitude & $74 \quad 146.14(74.235039)$ \\
\hline GPSAltitudeRef & Sea level \\
\hline GPSAltitude & $31.09 \mathrm{~m}$ \\
\hline GPST imeStamp & $1517 \quad 19.14$ \\
\hline GPSImgDirectionRef & Magnetic drection \\
\hline GPSImgDirection & 115.65 \\
\hline GPSD ateStamp & 2015:01:29 \\
\hline
\end{tabular}

Fig. 6. GPS EXIF information retrieved from an image.

\section{Backend: Database Server}

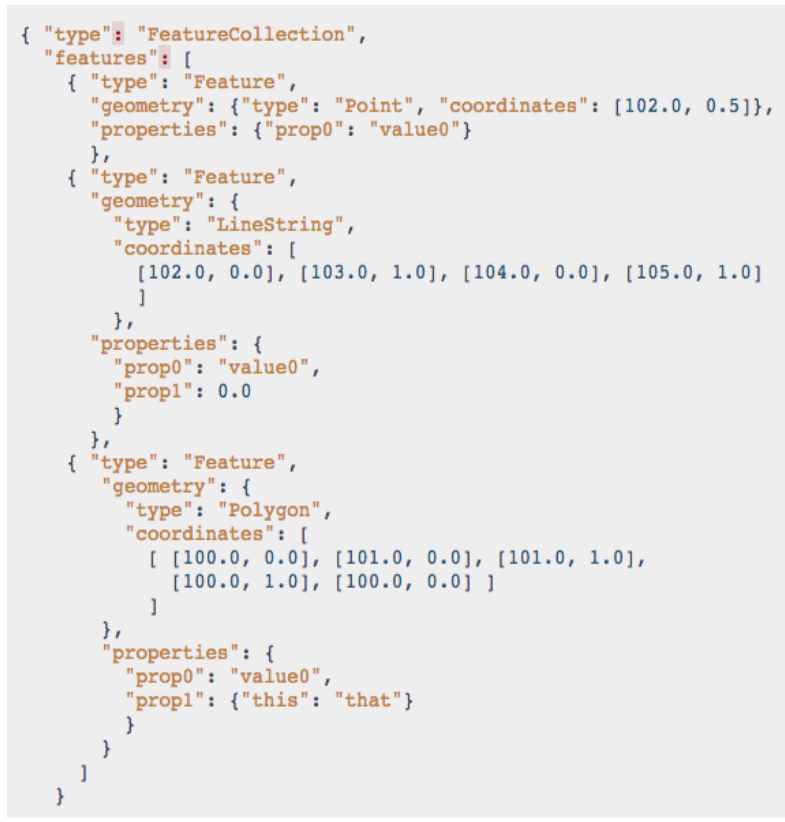

Fig. 7. JSON document with richer and more flexible data structures.

For this application, instead of using a relational database, MongoDB [9] will be used. Since the performance between a RDBMS and MongoDB is marginally in favor of MongoDB, it seems like the best choice for this case. The database server will receive a lot of requests when it is used in a large scale (ex. Open House), and being able to do queries fast will help in showing information on the application faster.

MongoDB structures data into collections of JavaScript Object Notation (JSON) [10] documents. JSON is a selfdescribing, human readable data format. Originally designed for lightweight exchanges between browser and server, it has become widely accepted for many types of applications. JSON is also a natural data format for use in the application layer [11]. Supporting JSON will enable to make more flexible data structures, as shown in Fig. 7.

\section{COURSE FRAMEWORK}

This independent study has several components that are necessary to develop the system. Some of them are not offered in regular courses so students have to work with faculty design their own curriculum. The framework includes the computer programming languages for designing the front-end and back-end, database knowledge for storing and retrieving the data, AR and GIS knowledge to work on the location and Google Map API, and the mobile app development skills to implement the system on mobile device.

\section{A. Component 1: Programming Language}

As this application is built using web technologies such as Javascript, HTML and CSS, classes such as web development greatly help understand how to build a frontend interface and how to effectively use frameworks to build more responsive and modern applications. As this application use JavaScript in the client and server side, a good foundation in the language in needed. 


\section{B. Component 2: Database}

Many students only have experience with relational databases with languages like MySQL, but as this study is using MongoDB, it is recommended to at least have some general database knowledge. Classes such as Database Management Systems have helped understanding how to manage a database and access rights for different users.

\section{Component 3: Augmented Reality}

To integrate the Augmented Reality element to the system, a combination between Google Maps and Unity engine will be implemented to show users directions to their desired location with their back-facing camera. Background using Unity engine is preferable, but Google Maps API knowledge is more desired.

\section{Component 4: Geographic Information System}

To get familiar and proficient with building a geographical information system, tools like ArcGIS were used to grasp the concept. Hackathon experience trying out these new concepts has improved the proficiency of building this system and overall understanding of the study.

\section{E. Component 5: Mobile Application Development}

Previous internship experience has expanded mobile app development knowledge. As many successful products has been implemented in mobile applications, the best way to make this application accessible to the general public is to make it available in an app.

\section{CONCLUSIONS}

As geobased applications are becoming popular and getting more exposure, a study like this shows how geolocation can be used to make systems that allow more immersive experiences with the surroundings around a person. This study has helped understand how geolocation works and how we can use places like buildings and build entities on top on them. As a student, this study greatly helps the understanding of how to build a geolocation service for consumers. As this is a current work in progress, the expectations for Spring semester is to fully bring this project to life and test a pilot with the Open House events to provide prospective students with an immersive experience of the campus.

\section{REFERENCES}

[1] N. Schuurman, "GIS: A short introduction," Wiley Publishing, May 2004.

[2] P. Bolstad, GIS Fundamentals: A First Text on Geographic Information Systems, XanEdu Publishing Inc., 2016.

[3] K. D. Pomfret, "Policy \& privacy: Geolocation - Lawmakers eye geolocation apps," GeoSpatial World, November 30, 2016.

[4] "Geolocation 101: How it works, the apps, and your privacy", PCWorld, Mar 29, 2010.

[5] J. Michael Sly, Getting around with Google Maps: A Programmer's Guide to the Google Maps API, CreateSpace Independent Publishing Platform, 2014.

[6] How Accurate is the GPS on my Smartphone? (Part 1). [Online]. Available:

https://communityhealthmaps.nlm.nih.gov/2014/06/30/how-accurateis-the-gps-on-my-smartphone/

[7] How Accurate is the GPS on my Smartphone? (Part 2). [Online]. Available:

https://communityhealthmaps.nlm.nih.gov/2014/07/07/how-accurateis-the-gps-on-my-smart-phone-part-2/

[8] E. Petroutsos, "Google maps: Power tools for maximizing the API," McGraw-Hill Education, 2014.

[9] K. Chodorow, "MongoDB: The definitive guide: Powerful and scalable data storage," O'Reilly Media, 2013.

[10] L. Bassett, "Introduction to javascript object notation: A to-the-point guide to JSON, " O'Reilly Media, 2015.

[11] G. Fourny, M. Brantner, and F. Cavalieri, "JSONiq: The SQL of NoSQL," CreateSpace Independent Publishing Platform, 2013.

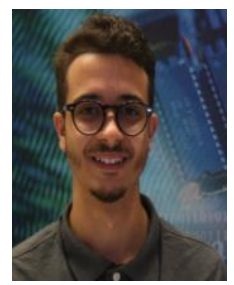

Jonathan Rodriguez was a senior student at Kean University in Union, New Jersey. And he graduated in 2017 with a BS degree in computer science. He is currently a software developer in the financial industry working on legacy applications and next generation architecture.

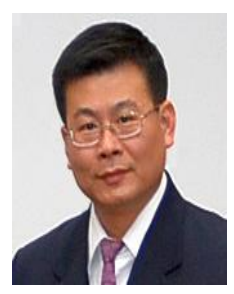

Ching-Yu Huang is an assistant professor of the School of Computer Science at Kean University since September 2014. Dr. Huang was born in 1968 in Taiwan and received a Ph.D. in computer \& information science from New Jersey Institute of Technology, Newark, New Jersey, USA in January 1998.

Prior to joining Kean University, Dr. Huang had more than 16 years' experience in the industry and academics in software development and $R \& D$ in bioinformatics. His research focuses SNP genotype calling and cluster detection; image processing and pattern recognition, especially in microarray and fingerprint; geotagged images and location information reconstruction; database application development; data processing automation; E-learning, educational multimedia, methodology, and online tools for secondary schools and colleges. Dr. Huang has more than 20 publications in journals and conferences and more than 20 presentations in workshops and invited lectures. 